\title{
Identification of Celiac Trunk Variations using Multi-Detector Computed Tomographic Angiography
}

Enam H. Moneeb ${ }^{1}$, Emad M. Nafie ${ }^{1}$, Radhiana Hassan ${ }^{2}$

${ }^{1}$ Department of Basic Medical Science, Kulliyyah of Medicine, International Islamic University Malaysia,

${ }^{2}$ Department of Radiology, Kulliyyah of Medicine, International Islamic University Malaysia.

Introduction: Variation in celiac trunk is common and has importance in preoperative planning to reduce the risk of accidental vascular injuries during surgical and radiological intervention. Our aim was to measure the prevalence of coeliac trunk types and describe its different variations using MDCT angiography among patients in Hospital Tengku Ampuan Afzan (HTAA), Kuantan. Materials and method: A retrospective cross sectional study was conducted. Computed Tomographic Angiography (CTA) images were retrieved from July till December 2017. Images were reconstructed in 3D Volume Rendering (VR) format using workstation and reviewed for the normal and anatomical variations of the celiac trunk. The variations were classified using Uflacker's classification. The results were calculated using IBM SPSS statistic version 22. Results: A total of 140 cases were found, from which 23 were excluded. Mean age was 57.3 years and male/female percentage was $65.8 / 34.2$. Anatomical variation of the CT was found in $54(46.2 \%)$ cases. The most common variation was the origin of inferior phrenic artery (IPA) from the CT in $21(17.9 \%)$ cases followed by celiac-colic trunk in $15(12.8 \%)$ cases. However, 31 (26.5\%) cases displayed variations not described in Uflacker's classification. They include celiaco-phrenic trunk, coexistence of IPA with other Uflacker's variations, Buhler arc, accessory hepatic artery from the CT and coexistence of celiac-colic trunk with gastro-splenic and hepato-splenic trunk in one case each. Conclusion: Accurate knowledge and identification of anatomical variations in CT is crucial before undergoing any surgical or invasive imaging procedure. This can help surgeons and interventional radiologists to prevent accidental vascular injuries and perform a safe procedure. 